\title{
Localisation of ventricular septal defects by simultaneous display of superimposed colour Doppler and cross sectional echocardiographic images
}

\author{
E ORTIZ, P J ROBINSON, J E DEANFIELD, R FRANKLIN, F J MACARTNEY, \\ R K H WYSE
}

From the Department of Paediatric Cardiology, Institute of Child Health, London

SUMMARY Precise non-invasive localisation of the site of a small ventricular septal defect was attempted using a new technique that simultaneously combines conventional cross sectional echocardiography with a Doppler system by superimposing the colour coded direction and velocity of blood flow directly on to real time ultrasound images. Twenty three patients with unoperated ventricular septal defects and a further eight after surgical closure were studied; 12 children with normal hearts served as controls. A colour coded blood flow jet entering the right ventricle during systole was identified in all 23 unoperated patients, in 11 of whom the defect was too small to be visualised by conventional cross sectional echocardiography. The colour Doppler technique precisely located 19 perimembranous and five trabecular defects (one patient had two defects). Five of the postoperative patients were without clinical evidence of a significant shunt but had pansystolic murmurs. In each of these five, trans-septal shunt blood flow was demonstrated by colour Doppler images whereas in only three of these patients was the residual defect large enough to be visualised by conventional cross sectional echocardiography. Three postoperative patients had no murmurs and showed no residual shunt on colour Doppler images. This was confirmed at cardiac catheterisation. There were no false positive results among the controls.

This technique is useful for the more accurate diagnosis and location of ventricular septal defects and may help in assessing their natural or surgical closure.

Despite recent advances in ultrasound instrumentation and image quality, difficulties may be encountered in identifying small ventricular septal defects, particularly within the trabecular septum. ${ }^{1-3}$ There remains a sizeable number of patients in whom the diagnosis of ventricular septal defect is based solely on clinical findings such as the character and location of the systolic murmur. Consequently, this study aimed to determine if diagnosis could be improved by the detection of trans-septal blood flow in patients with small ventricular septal defects using a new colour coded Doppler system that superimposes flow information on to conventional cross sectional images

Requests for reprints to Dr R K H Wyse, Department of Paediatric Cardiology, Institute of Child Health, 30 Guilford Street, London WCIN IEH.

Accepted for publication 5 February 1985 (colour Doppler). This system has already proved useful in assessing acquired valvar diseases, particularly regurgitation. 45 With its unique display, we reasoned that this system should be able precisely to locate ventricular septal defects in a way hitherto impossible, and thus we considered it to be important in the present study to assess the clinical usefulness of this novel approach.

\section{Patients and methods}

\section{STUDY GROUPS}

This work formed part of a comprehensive longitudinal follow up study on a group of 182 patients with ventricular septal defects conducted from June 1980 to August 1984 at the Hospital for Sick Children, London. Thirty one patients were randomly selected to constitute the study group.

The patients were subsequently divided into three groups. Group 1 consisted of 12 patients (age range 90 
Ortiz, Robinson, Deanfield, Franklin, Macartney, Wyse

days to 12.3 years; median 2.5 years) whose ventricular septal defect had previously been visualised and anatomically located by conventional cross sectional echocardiography (Advanced Technology Laboratory mechanical sector scanner, model 600). One patient in this group also had pulmonary stenosis with a valve gradient of $45 \mathrm{~mm} \mathrm{Hg}$ and peak right and left ventricular systolic pressures of $70 \mathrm{~mm} \mathrm{Hg}$ and $86 \mathrm{~mm}$ $\mathrm{Hg}$ respectively. Group 2 consisted of 11 patients (age range 96 days to $15 \cdot 1$ years; median 4.3 years) whose ventricular septal defect had been diagnosed clinically but was too small to be visualised by conventional cross sectional echocardiography. Group 3 consisted of eight postoperative patients who had undergone surgical closure of their ventricular septal defects (age range 78 days to 6.75 years; median 2.6 years; mean time elapsed since surgery 5.9 months). A separate group of 12 children (age range 60 days to 10.5 years; median 5.6 years) with normal hearts served as controls.

\section{ECHOCARDIOGRAPHY}

The combined cross sectional and colour coded Doppler echocardiographic studies were performed by the operator before any physical examination or auscultation was carried out. Doppler signals, colour coded for the direction of blood flow, were obained using an Aloka SSD-880 ultrasound scanner. This scanner, by incorporating a recently developed autocorrelator, ${ }^{6}$ encodes blood flow in terms of colour and instantaneously superimposes this information in real time across either the entire screen of a standard cross sectional echocardiographic display or a selected region. Colours were assigned in accordance with the direction of blood flow; blue was arbitrarily given to blood flowing towards the transducer, while red was given to the blood flowing away from it. Colour brightness, displayed in seven gradations, was proportional to the velocity of blood flow. The emitting frequency used was 2.5 or $3.5 \mathrm{MHz}$ as appropriate. Real time signals were stored using a Sony SLO-420 Betamax video tape recorder and later transferred to a Sony U-matic format. These images were replayed on a Sony VO5800PS recorder through a colour monitor, either at normal speed or in slow motion as required. Still frames were taken either at the time of study from an integral Polaroid camera or subsequently by photographing images frozen by the stop frame facility on the U-matic recorder directly from the monitor.

As a screening procedure in all patients, the transducer was positioned to obtain good views of the ventricular septum in all planes and axes of the heart as previously described. ${ }^{2}$ Particular attention was given to the four chamber, long axis, and short axis views from the apical and parasternal approaches.

\section{Results}

Studies of the 12 control children without any cardiac abnormalities allowed the normal pattern of colour coded blood flow in the heart to be identified (Fig. 1). The presence of ventricular septal defect flow detected in the right ventricle of patients was easily distinguished from the normal pattern of right ventricular flow seen in the controls.

A colour coded blood flow jet entering the right ventricle during systole was identified in all 23 patients with unoperated ventricular septal defects irrespective of whether the defect could be seen in the septum (groups 1 and 2) (Fig. 2). In group 1 patients the ventricular septal defect was defined on cross sectional echocardiography and verified by colour Doppler images to be perimembranous in nine and trabecular in three (two central, one apical). In group 2 blood flow jets entering the right ventricle were seen in a perimembranous position in nine patients and in a central trabecular position in one (Fig. 3). In another patient, previously thought clinically to have a perimembranous ventricular septal defect, the blood flow jet was clearly seen emanating from the central muscular area with the colour Doppler technique. Another patient in group 2 showed two separate jets in the perimembranous and trabecular areas of the septum at the same time, the latter being subsequently recognised anatomically by cross sectional echocardiography once its precise location had been demonstrated by the colour coded blood flow jet. Indeed similar post-colour Doppler anatomícal recognition of ventricular septal defects by conventional cross sectional echocardiography occurred in three more patients in group 2. Figure 4 shows composite findings obtained from a patient in group 1 with both ventricular septal defect and pulmonary stenosis showing that the colour Doppler technique was able clearly to visualise blood flow across the defect even in the presence of a raised right ventricular peak systolic pressure (pulmonary valve gradient $45 \mathrm{~mm} \mathrm{Hg}$ at cardiac catheterisation).

In all eight postoperative patients (group 3 ) the patch used to close the ventricular septal defect could be seen on standard cross sectional echocardiography, and residual defects were visualised in three of these. Systolic murmurs were audible in these three and in two other patients. Systolic flow jets emanating from the patch site were seen in the right ventricle of all five of these patients with systolic murmurs (Fig. 5). In the three postoperative patients without systolic murmurs no blood flow jets indicative of a ventricular septal defect were seen on colour Doppler images (Fig. 6). The absence of any residual defect in each of these three patients was also confirmed at cardiac catheterisation. 

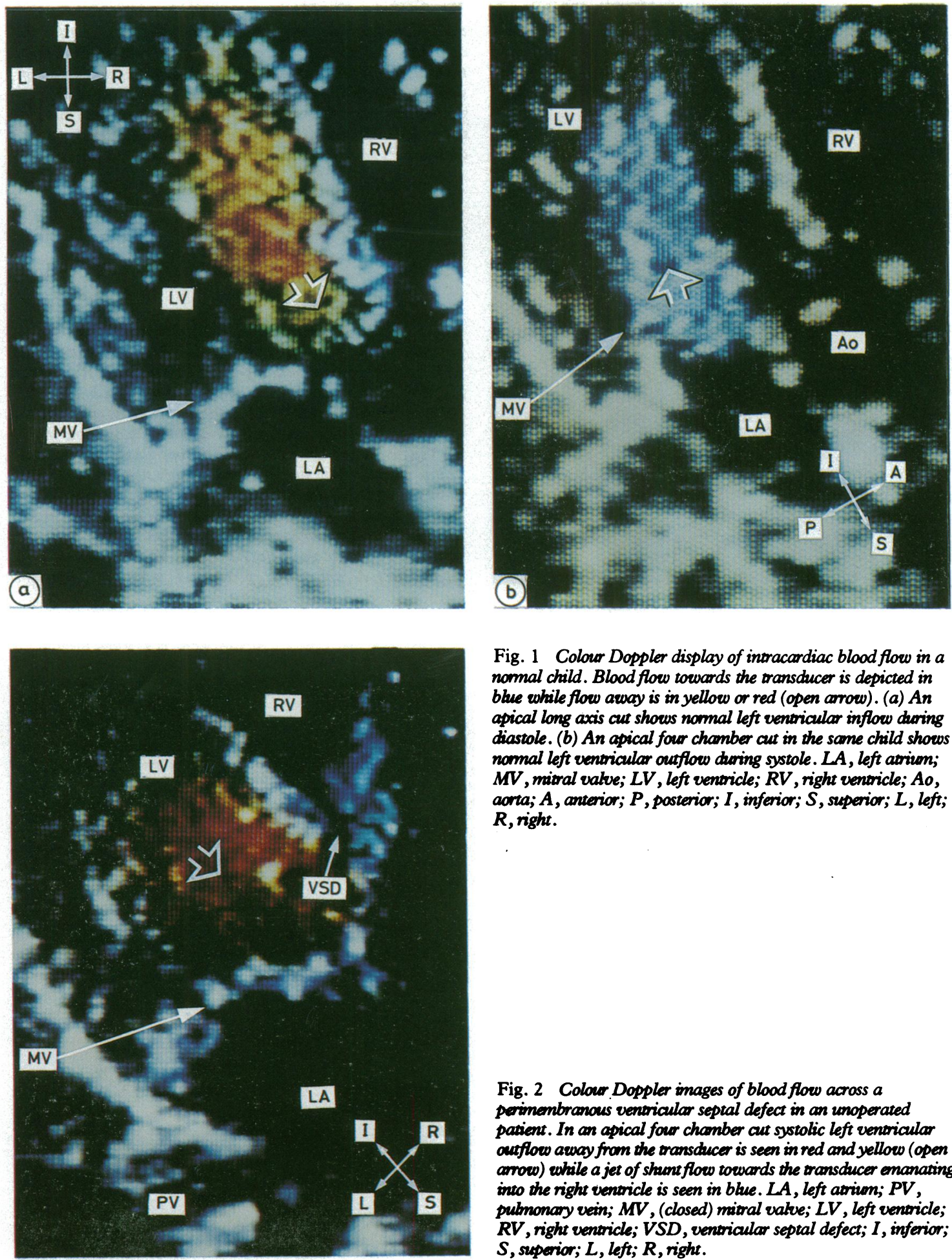

Fig. 1 Colour Doppler display of intracardiac blood flow in a normal child. Blood flow towards the transducer is depicted in blue while flow away is in yellow or red (open arrow). (a) An apical long axis cut shows nomal left ventricular inflow dearing diastole. (b) An apical four chamber cut in the same child shows nomal left ventricular outflow during systole. LA, left atrivon; $M V$, mitral valve; $L V$, left ventricle; $R V$, right ventricle; $A 0$, aorta; $A$, anterior; $P$, posterior; $I$, inferior; $S$, superior; $L$, left; $R$, right.

Fig. 2 Colow Doppler images of blood flow across a perimembranous ventricular septal defect in an unoperated pasient. In an apical four chamber cut systolic left ventricular outflow away from the transducer is seen in red and yellow (open arrow) while a jet of shumt flow towards the transducer emanating into the right ventricle is seen in blue. LA, left atrium; $P V$, pulmonary vein; $M V$, (closed) mitral valve; $L V$, left ventricle; $R V$, right ventricle; VSD, ventricular septal defect; $I$, inferior; $S$, superior; $L$, left; $R$, right. 

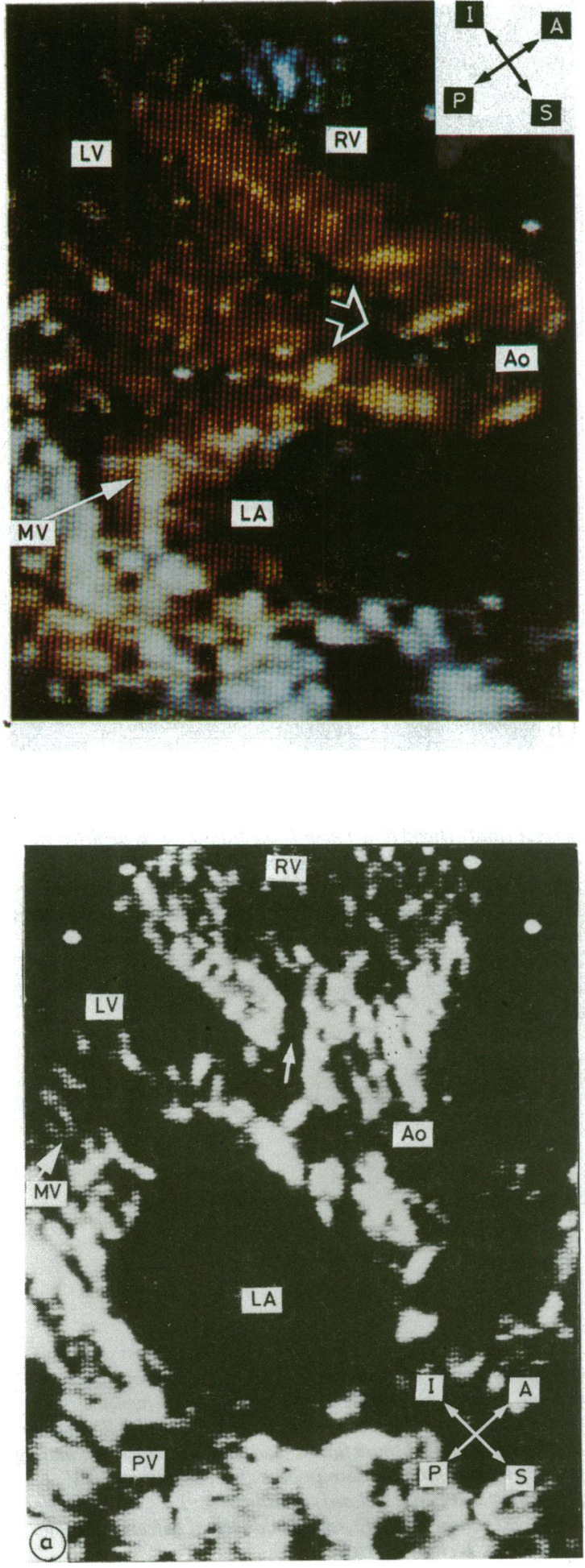

Fig. 3 Colow Doppler image of blood flow across a central trabecular ventricular septal defect in an umoperated patient. In an apical long axis cut left ventricular outflow away from the transducer is seen in red and yellow (open arrow) while a jet of shant flow towards the transducer emanating into the right ventricle is seen in blue. LA, left atriven; $M V$, (closed) mitral valve; $L V$, left ventricle; $R V$, right ventricle; Ao, aorta; $A$, anterior; $P$, posterior; $I$, inferior; $S$, superior.

Fig. 4 Colour Doppler images of blood flow across a perimembranous ventricular septal defect in an unoperated child with a raised right ventricular peak systolic pressure $(R V, 70 \mathrm{~mm}$ $\mathrm{Hg}$; LV, $85 \mathrm{~mm} \mathrm{Hg}$ ) due to pulmonary stenosis (pulmonary valve gradient $45 \mathrm{~mm} \mathrm{Hg}$ ). (a) An apical long axis cut shows the location of the defect. (b) A similar cut shows blood flow in the left ventricular outflow tract in systole (open arrow) in red and yellow while, despite significant pulmonary stenosis, a jet of shuont flow towards the transducer may be clearly seen in blue emanating into the right ventricle at the site of the defect. $L A$, left atrivm; $P V$, pulmonary vein; $M V$, mitral valve; $L V$, left ventricle; $R V$, right ventricle; $A o$, aorta; $V S D$, ventricular septal defect; $A$, anterior; $P$, posterior; $I$, inferior; $S$, superior.

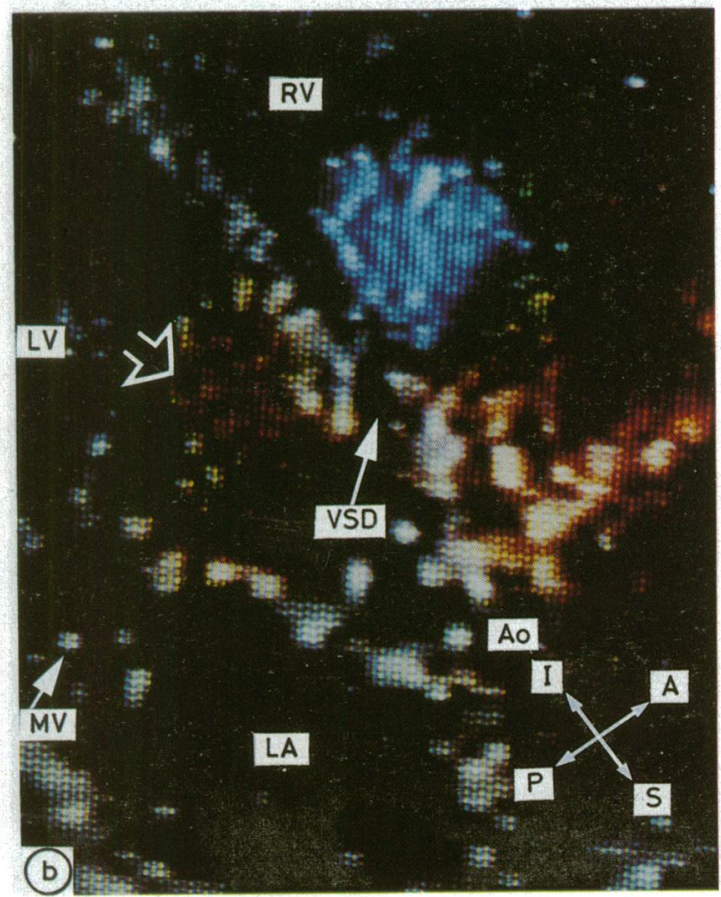



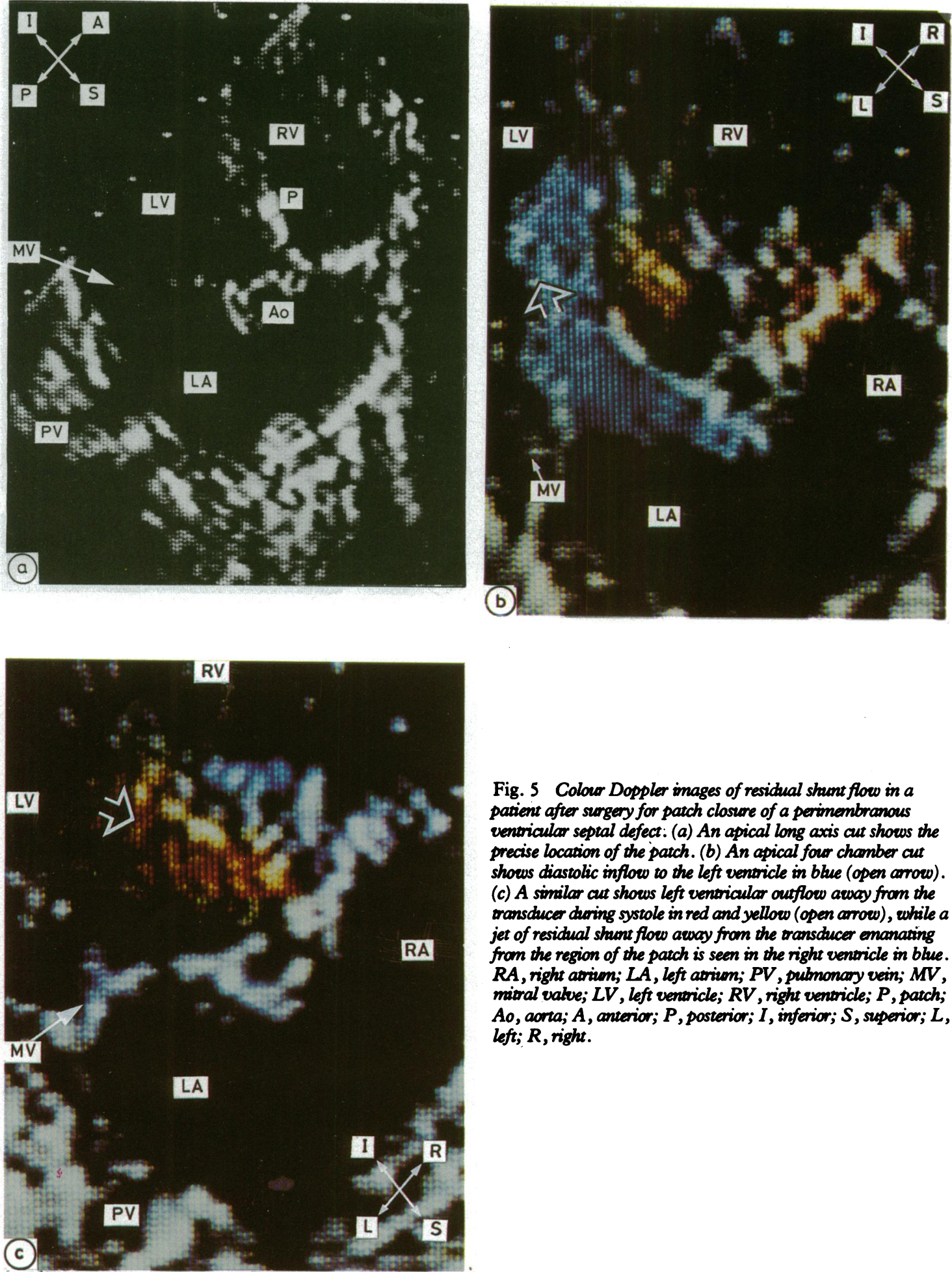

Fig. 5 Colour Doppler images of residual shumt flow in a patient after surgery for patch closure of a perimembranous ventricular septal defect: (a) An apical long axis cut shows the precise location of the patch. (b) An apical four chamber cut shows diastolic inflow to the left ventricle in blue (open arrow). (c) A similar cut shows lefi ventricular outflow arvay from the transducer during systole in red and yellow (open arrow), while a jet of residual shumt flow awday from the transchucer emanating from the region of the patch is seen in the right ventricle in blue. $R A$, right atrivom; $L A$, left atriven; $P V$, pulmonary vein; $M V$, mitral valve; $L V$, lefi ventricle; $R V$, right ventricle; $P$, patch; Ao, aorta; $A$, anterior; $P$, posterior; $I$, inferior; $S$, superior; $L$, lef; $R$, right. 

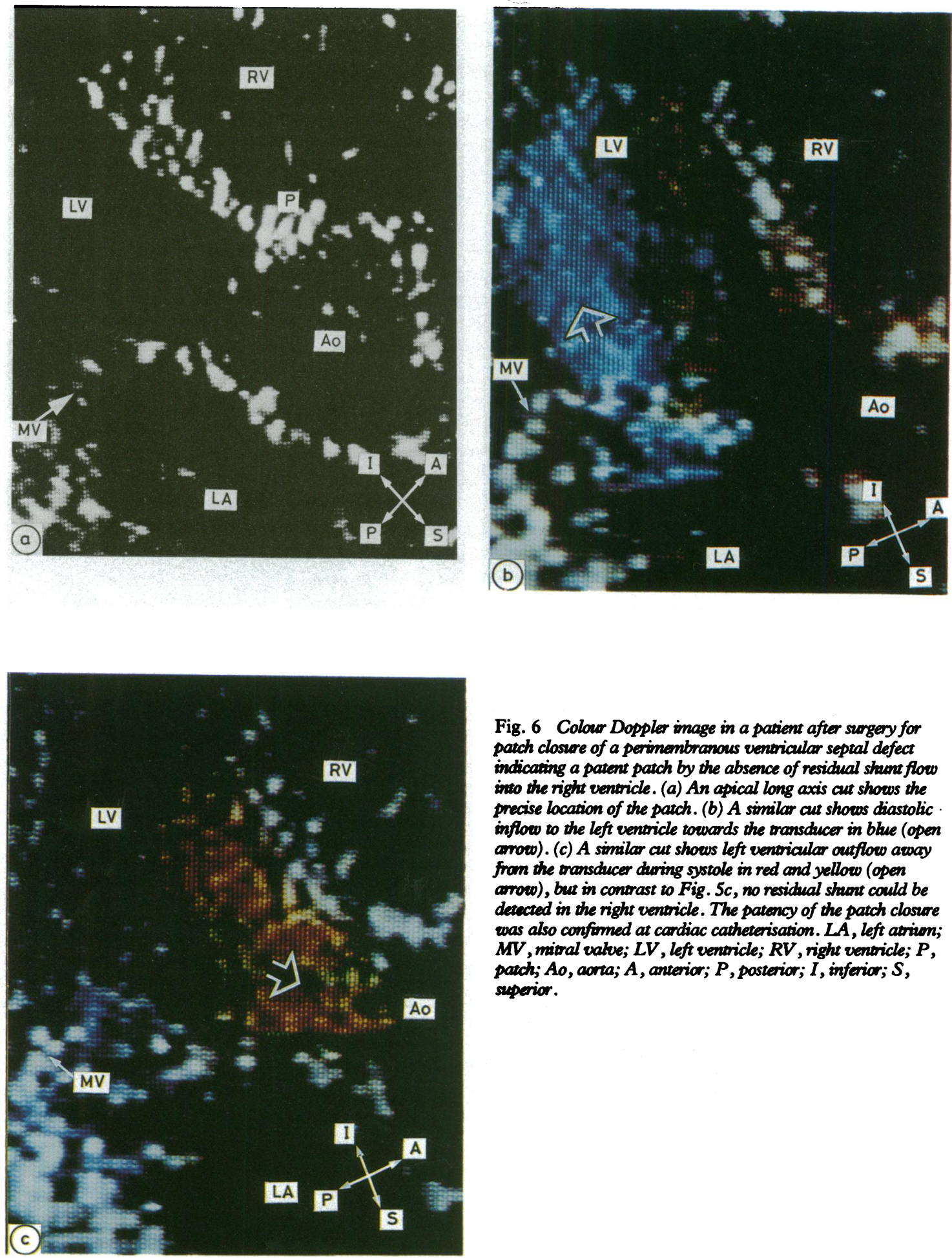

Fig. 6 Colour Doppler image in a patient after surgery for patch closure of a perimembranous ventricular septal defect indicating a patent patch by the absence of residual shemt flow into the right ventricle. (a) An apical long axis cut shows the precise location of the patch. (b) A similar cut showes diastolic inflow to the left ventricle towards the transducer in blue (open arrow). (c) A similar cut shows left ventricular outflow away from the transducer dering systole in red and yellow (open arrow), but in contrast to Fig. $5 c$, no residual shomt could be detected in the right ventricle. The patency of the patch closure was also confirmed at cardiac catheterisation. $L A$, left atrivon; $M V$, mitral valve; $L V$, left ventricle; $R V$, right ventricle; $P$, patch; $A 0$, aorta; $A$, anterior; $P$, posterior; $I$, inferior; $S$, superior. 


\section{Discussion}

Cross sectional echocardiography can visualise a ventricular septal defect only if the defect size is more than the resolution of the ultrasound scanner (normally approximately $2 \mathrm{~mm}$ ). Problems in diagnosis are, however, compounded if the defect is located in the trabecular septum or there are multiple defects. In trying to overcome these difficulties, an approach allowing the direct visualisation of blood flow across the ventricular septum seems ideal. Until now, demonstration of trans-septal flow in small ventricular septal defects could be reliably achieved only by cardiac catheterisation and angiocardiography. Nevertheless, invasive techniques carry a significant risk of morbidity and mortality, and clearly a reliable non-invasive alternative would be preferable. Several non-invasive Doppler techniques for detecting ventricular septal defects have been reported. ${ }^{7-13}$ Jugular venous continuous wave Doppler techniques are unreliable particularly with smaller defects, and there are also several disadvantages of methods using standard pulsed Doppler ultrasound. Using this technique, Magherini et al were unable to localise defects in the inlet portion and found ventricular septal defects difficult to detect if the shunt was balanced as in pulmonary hypertension. ${ }^{11}$ Furthermore, ventricular septal defects could not be differentiated from right ventricular outflow tract obstruction. When a small pulsed Doppler sample volume is used as a roving interrogator of flow within the ventricular cavities and septum, it is never certain whether absence of detected systolic turbulence means that there is no defect or that a jet has been missed. The colour Doppler technique differs from previous approaches in that flow within an entire echocardiographic cross sectional cut of the heart is simultaneously displayed, thus minimising the possibility of missing the blood flow jet of a ventricular septal defect. Furthermore, the diagnosis is based on a combination of blood flow turbulence, direction, and timing.

In the present study, we found that using the colour Doppler technique the presence of trans-septal blood flow was clearly demonstrated in every patient with a ventricular septal defect. Furthermore, the technique permitted detection and localisation of ventricular septal defects in patients whose defects were too small to be visualised by standard cross sectional echocardiography. The recent findings of Miyatake $e t \mathrm{al}^{5}$ concur with this result. Using a short axis view in a patient with a small ventricular septal defect they also found that colour Doppler images clearly demonstrated the flow disturbance even though conventional cross sectional echocardiography was unable to detect the defect. Though our study was not designed to determine accurately the specificity and sensitivity of the method, both are clearly superior to standard cross sectional echocardiography. The technique also proved of use in the case of multiple ventricular defects by simultaneous demonstration of more than one flow jet. Similarly, it was shown to be useful in demonstrating the presence or absence of any residual ventricular septal defect in patients after primary surgical closure. The need to look serially for the presence of residual ventricular septal defects in patients in the immediate postoperative period has been shown by Stevenson et al. ${ }^{14}$ Immediately after surgery they found a $93 \%$ incidence of residual shunts, a value which decreased progressively over the ensuing days. It seems likely that the colour Doppler technique will be able to simplify the initial detection, localisation, and serial monitoring of these residual defects.

Many of the patients in this study had heart rates of over 100 beats/minute. In all patients, but particularly in these, it was necessary to review blood flow information in slow motion in order definitively to locate trans-septal flow in the right ventricle in systole. This is because in most patients, using an apical four chamber view, the trans-septal blood flow jet was predominantly towards the transducer. Since diastolic inflow is in the same direction it also produced a similar colour Doppler appearance within the right ventricle. Thus, by replaying the colour Doppler recording in slow or stopped motion, the transseptal blood flow jet depicted in blue could be seen frozen against a red background of ventricular ejection. In the same stopped frame, systole could be confirmed by a Doppler shift to the red within the left ventricular outflow tract. Thus any confusion with diastolic inflow that could occur in real time was easily avoided.

We conclude that direct visualisation of trans-septal blood flow by colour Doppler is reliable and reproducible and will provide clinical help in excluding ventricular septal defects in patients with innocent murmurs and in those whose ventricular septal defects are thought to have been surgically closed. Most importantly, the technique permits accurate localisation of defects that are otherwise invisible to cross sectional echocardiography. Since there is increasing evidence of the key role of the site of the defect to its natural history and probability of spontaneous closure, ${ }^{15}$ the method should in the future help in providing more accurate prognostic information for the individual patient.

FJM and RKHW are supported by the Vandervell and British Heart Foundations. This work was supported in part by the Child Health Research Appeal Trust. 


\section{References}

1 Bierman FZ, Fellows K, Williams RG. Prospective identification of ventricular septal defects in infancy using subxiphoid two-dimensional echocardiography. Circulation 1980; 62: 807-17.

2 Sutherland GR, Godman MJ, Smallhorn JF, Guiterras P, Anderson RH, Hunter S. Ventricular septal defects. Two dimensional echocardiographic and morphological correlations. Br Heart $\mathcal{f}$ 1982; 47: 316-28.

3 Capelli H, Andrade JL, Somerville J. Classification of the site of ventricular septal defect by 2-dimensional echocardiography. Am $\mathcal{f}$ Cardiol 1983; 51: 1474-80.

4 Omoto R, Yokote Y, Takamoto S, et al. The development of real-time two-dimensional Doppler echocardiography and its clinical significance in acquired valvular diseases: with special reference to the evaluation of valvular regurgitation. F pn Hear f 1984; 25: 325-40.

5 Miyatake K, Okamoto $M$, Kinoshita N, et al. Clinical applications of a new type of real-time two-dimensional Doppler flow imaging system. Am $\mathcal{F}$ Cardiol 1984; 54: 857-68.

6 Namekawa K, Kasai C, Tsukamoto M, Koyano A. Imaging of blood flow using autocorrelation [Abstract]. Ulmasound Med Biol 1982; 8: 138.

7 Kalmanson DJ, Aigueperse J, Veyrat C, Cornec C, Chiche $P$. Non-invasive technique for diagnosing congenital and acquired ventricular septal defects using directional Doppler ultrasound: correlations in phasic flow velocity patterns of shunt. Br Heart $\mathcal{F} 1974$; 36: $428-45$.
8 Johnson SL, Baker DW, Lute RA, Kawabori I. Detection of small ventricular septal defects by Doppler flowmeter [Abstract]. Circulation 1974; 50 (suppl III): 142.

9 Stevenson JG, Kawabori I, Guntheroth WG. Differentiation of ventricular septal defects from mitral regurgitation by pulsed Doppler echocardiography. Circulation 1977; 56: 14-8.

10 Stevenson JG, Kawabori I, Dooley T, Guntheroth WG. Diagnosis of ventricular septal defect by pulsed Doppler echocardiography: sensitivity, specificity and limitations. Circulation 1978; 58: 322-6.

11 Magherini A, Azzolina V, Wiechmann V, Fantini F. Pulsed Doppler echocardiography for the diagnosis of ventricular septal defects. Br Heart F 1980; 43: 143-7.

12 Colo J, Stevenson JG, Pearlman AS. A comparison of two dimensional echocardiography and pulsed Doppler for diagnosis of ventricular septal defect [Abstract]. Circulation 1982; 66 (suppl II): 232.

13 Murphy DJ Jr, Meyer RA, Kaplan S. 2D contrast echo and pulsed Doppler to assess residual ventricular shunts in the immediate post-operative period [Abstract]. Pediat Res 1983; 17: 118A.

14 Stevenson JG, Kawabori I, Stamm SJ, et al. Pulsed Doppler echocardiographic evaluation of ventricular septal defect patches. Circulation 1984; 70 (suppl I): 38-46.

15 Sutherland GR, Bain HH, Anderson RH, Hunter S. Natural history of ventricular septal defects-long term prospective two dimensional echocardiographic study [Abstract]. Br Heart F 1983; 49: 293P-4P. 